Series A

\author{
I. MATHEMATICA
}

447

\title{
DEGREE AND POINT-INVERSES OF MAPPINGS ON SPHERES
}

BY

MATTI HONKAPOHJA

HEL S I K I 1969

S U O M A L A I EN T IEDEAKATEM I A

doi:10.5186/aasfm.1969.447 
Communicated 14 March 1969 by P. J. Myrberg and Olli Lehto 


\section{Degree and point-inverses of mappings on spheres}

The purpose of this paper is to prove a theorem suggested by considerations in [1], p. 270. Although the theorem has some obvious generalizations (see theorem 2), we shall prove the following original form of it:

Theorem 1. If $f$ is a continuous mapping of the $n$-sphere $S^{n}$ into $S^{n}$ and if $|\operatorname{deg}(f)|=k>0$, then the set of points $y \in S^{n}$ for which $f^{-1}(y)$ contains at least $k$ points is dense in $S^{n}$.

In what follows, $f$ will always mean a continuous mapping of $S^{n}$ into $S^{n}$. For a point $y \in S^{n}$, we call $f^{-1}(y)$ the point-inverse of $y$. By a domain we shall mean an open, non-empty, connected subset of $S^{n}$. The letter $D$ stands as a symbol for a domain. A neighbourhood of a point $x \in S^{n}$, denoted by $U(x)$, is also always assumed to be a domain. The boundary of a set $A \subset S^{n}$ is denoted by $\partial A$.

The (local) degree (topological index) of a map $f$ is an integer-valued function of triples $(f, D, y)$ where $D \subset S^{n}$ is a domain and $y \in S^{n}-f(\partial D)$. For such a triple the value of the degree is denoted by $\operatorname{deg}(f, D, y)$. We shall need the following properties of the degree (for the definition of the degree and the proofs of the properties see e.g. [1], [2]):

(1) $\operatorname{deg}(f, D, y)=\operatorname{deg}\left(f, D, y^{\prime}\right)$, if $y$ and $y^{\prime}$ belong to the same component of $S^{n}-f(\partial D)$.

(2) If $\operatorname{deg}(f, D, y) \neq 0$, then $y \in f(D)$.

(3) If $f \mid \bar{D}$ is injective and if $y \in f(D)$, then $|\operatorname{deg}(f, D, y)|=1$.

(4) Let $D_{1}, \ldots, D_{k} \subset D$ be disjoint and let $y \in S^{n}-f(\partial D)$ such that $D \cap f^{-1}(y) \subset \bigcup_{i=1} D_{i}$. Then

$$
\operatorname{deg}(f, D, y)=\sum_{i=1}^{k} \operatorname{deg}\left(f, D_{i}, y\right)
$$

From (1) it follows that $\operatorname{deg}\left(f, S^{n}, y\right)$ has the same value for all $y \in S^{n}$. This common value is the global degree of $f$, denoted by $\operatorname{deg}(f)$.

For the sake of convenience, we shall make the following additional definition:

Let $f$ be a map, $x \in S^{n}$ and $y=f(x)$. We call $x$ an essential point of $f$ if $x$ has a neighbourhood $U(x)$ such that $\overline{U(x)} \cap f^{-1}(y)=\{x\}$ and $\operatorname{deg}(f, U(x), y) \neq 0$. 
Remark 1. Suppose that $x$ is an essential point of $f$ and that $U_{1}(x)$ and $L_{2}(x)$ are neighbourhoods of $x$ such that $\overline{U_{i}(x)} \cap f^{-1}(f(x))=\{x\}$. Choose a neighbourhood $U_{3}(x) \subset U_{1}(x) \cap U_{2}(x)$. Then (4) implies that

$$
\operatorname{deg}\left(f, U_{1}(x), y\right)=\operatorname{deg}\left(f, U_{3}(x), y\right)=\operatorname{deg}\left(f, U_{2}(x), y\right),
$$

where $y=f(x)$. Thus $\operatorname{deg}(f, U(x), y)$ has the same value for all $U(x)$ such that that $\overline{U(x)} \cap f^{-1}(y)=\{x\}$.

Remark 2. Suppose that $D \subset S^{n}, y \in S^{n}-f(\partial D)$ and $\operatorname{deg}(f, D, y) \neq 0$. Assume further that $f^{-1}(y) \cap D$ (which is non-empty by (2)) contains only a finite number of points, say $x_{1}, \ldots, x_{k}$. Choose disjoint neighbourhoods $U\left(x_{i}\right) \subset D, i=1, \ldots, k$. (4) implies that

$$
0 \neq \operatorname{deg}(f, D, y)=\sum_{i=1}^{k} \operatorname{deg}\left(f, U\left(x_{i}\right), y\right) \text {. }
$$

Thus there must be essential points in $f^{-1}(y) \cap D$. Moreover, we have

$$
\operatorname{deg}(f, D, y)=\sum_{j=1}^{h} \operatorname{deg}\left(f, U\left(x_{i_{j}}\right), y\right)
$$

where $x_{i_{1}}, \ldots, x_{i_{h}}$ are the essential points of $f^{-1}(y) \cap D$.

For the proof of theorem 1 we still need a lemma:

Lemma. Let $f$ be a map, $D \subset S^{n}, y_{0} \in f(D)-f(\partial D)$, and let $U\left(y_{0}\right)$ $\subset f(D)-f(\partial D)$ be such that for every $y \in U\left(y_{0}\right), f^{-1}(y) \cap D$ is finite and contains exactly one essential point. Then

$$
\left|\operatorname{deg}\left(f, D, y_{0}\right)\right|=1 .
$$

Proof. Define a map $g: U\left(y_{0}\right) \rightarrow D$ by sending every $y \in U\left(y_{0}\right)$ to the essential point of $f^{-1}(y) \cap D$. We shall show that $g$ is continuous. For this purpose, let $y \in U\left(y_{0}\right), x=g(y)$, and let $U(x) \subset D$ be a neighbourhood of $x$, of which we may assume that $\overline{U(x)} \cap f^{-1}(y)=\{x\}$. Choose $U(y) \subset C^{r}\left(y_{0}\right)-f(\partial U(x))$. Then (1) implies that for any $y^{\prime} \in U(y)$,

$$
\operatorname{deg}\left(f, U(x), y^{\prime}\right)=\operatorname{deg}(f, U(x), y) \neq 0 .
$$

But this means that the essential point of $f^{-1}\left(y^{\prime}\right) \cap D$ lies in $U(x)$. Thus $g(U(y)) \subset U(x)$, which proves the continuity of $g$.

Since $g$ is continuous and certainly also injective, $g\left(U\left(y_{0}\right)\right)$ is open. Consequently, $x_{0}=g\left(y_{0}\right)$ has a neighbourhood $U\left(x_{0}\right)$ such that $\overline{U\left(x_{0}\right)}$ $\subset g\left(U\left(y_{0}\right)\right)$. Then $f \overline{U\left(x_{0}\right)}$ is injective, and by (3) and remark 2 we have

$$
\left|\operatorname{deg}\left(f, D, y_{0}\right)\right|=\left|\operatorname{deg}\left(f, U\left(x_{0}\right), y_{0}\right)\right|=1 \text {. }
$$

The proof of theorem 1. Suppose that there exists an open, non-empty set $B \subset S^{n}$, such that the point-inverse of any point of $B$ has less than 
$k$ points. By remark 2, $f^{-1}(y)$ contains essential points when $y \in B$. Take a point $y_{0} \in B$, for which $f^{-1}\left(y_{0}\right)$ contains a maximal number $m(<k)$ of essential points, say $x_{1}, \ldots, x_{m}$. Choose disjoint neighbourhoods $U\left(x_{i}\right)$, $i=1, \ldots, m$ such that $f^{-1}\left(y_{0}\right) \cap \overline{U\left(x_{i}\right)}=\left\{x_{i}\right\}, i=1, \ldots, m$. Then $y_{0}$ has a neighbourhood $U\left(y_{0}\right) \subset B$ such that for any $y \in U\left(y_{0}\right)$ we have

$$
\operatorname{deg}\left(f, U\left(x_{i}\right), y\right)=\operatorname{deg}\left(f, U\left(x_{i}\right), y_{0}\right) \neq 0,
$$

$i=1, \ldots, m$. This means that when $y \in U\left(y_{0}\right), f^{-1}(y) \cap U\left(x_{i}\right)$ contains essential points for all $i=1, \ldots, m$. But since the number of them cannot exceed $m$ there is exactly one of them in each $U\left(x_{i}\right)$. The preceding lemma and remark 2 then yield

$$
|\operatorname{deg}(f)|=\left|\sum_{i=1}^{m} \operatorname{deg}\left(f, U\left(x_{i}\right), y_{0}\right)\right| \leq \sum_{i=1}^{m}\left|\operatorname{deg}\left(f, U\left(x_{i}\right), y_{0}\right)\right|=m<k .
$$

This contradiction proves the theorem.

Theorem 1 can be generalized at least to $n$-dimensional orientable manifolds, for which degree theory can also be defined (see e.g. [3]):

Theorem 2. Suppose that $X$ and $Y$ are oriented n-manifolds and that $f: \bar{D} \rightarrow Y$ is a continuous mapping, where $D$ is a relatively compact domain of $X$. Let $y_{0} \in Y-f(\partial D)$, and let $C$ be the component of $Y-f(\partial D)$ containing $y_{0}$. If $\operatorname{deg}\left(f, D, y_{0}\right) \mid=k>0$, then the set of points $y \in C$, for which $f^{-1}(y)$ contains at least $k$ points, is dense in $C$.

The proof of the theorem is completely analogous to the one given above.

\section{References}

[1] Hocking, J. G. - Yotsg, G. S.: Topology. - Addison-Wesley; Reading, Mass. 1961.

[2] Rado, T. - Reichelderfer, P. V.: Continuous transformations in analysis. Springer; Berlin 1955.

[3] VärsäLä, J.: Discrete open mappings on manifolds. - Ann. Acad. Sci. Fenn. A I 392 (1966), $1-10$. 